\title{
Los principios de ISO 26000 como eje articulador del sistema integrado HSEQ - RUC en empresas consultoras en ingeniería y medio ambiente de Bogotá"
}

\author{
The principles of ISO 26000 as the linchpin \\ of integrated HSEQ - RUC system in consulting engineering \\ and environmental companies in Bogota
}

Recibido: 11 de febrero de 2014

Revisado: 14 de abril de 2014 Aceptado: 27de junio de 2014

Cristina Losada Sáenz***

Ministerio de Educación Nacional

Juan Carlos Carranza Martine: ****

Antea Group

\section{RESUMEN}

Empresas del sector de la consultoría en ingeniería y medio ambiente, objeto de este estudio, han implementado sistemas de gestión motivados por la necesidad de cumplir con un requisito contractual exigido por las empresas operadoras del sector petrolero, más que por garantizar una excelente gestión en sus procesos. Esta investigación define una propuesta metodológica para un modelo de gestión integral en empresas consultoras que prestan sus servicios a empresas pertenecientes al subsector de explotación de petróleo en Colombia, para articular

* Artículo de investigación

** Administradora de Empresas. Especialista en Administración y Gerencia de Sistemas de la Calidad. Profesional Especializado Ministerio de Educación Nacional. Correo electrónico: cristinalosadasaenz@yahoo.es

***Administrador de Empresas. Especialista en Administración y Gerencia de Sistemas de la Calidad. Director HSEQ. Correo electrónico: jkcarranza2000@yahoo.com 
adecuadamente los sistemas integrados de gestión Health, Safety, Environment, Quality - HSEQ. utilizando como eje articulador los principios de responsabilidad social contenidos en la Guía ISO 26000. Se realiza un despliegue de las hipótesis en variables y elementos por indagar. A partir de los principios de ISO 26000 se diseña una encuesta y se elabora la propuesta metodológica, ésta última con tres componentes: estratégico, técnico/operativo y humano. Para garantizar la validez de los resultados de la investigación se utiliza la triangulación de datos. Finalmente, se concluye que los principios de ISO 26000 son elementos articuladores de los componentes de la propuesta metodológica. Al ser esta una investigación aplicada, sus resultados pueden implementarse en otras organizaciones con sistemas integrados de gestión HSEQ y RUC.

Palabras clave: Gestión Integral, HSEQ, RUC, responsabilidad social empresarial, sistemas de gestión integrados, ISO 26000.

\section{ABSTRACT}

Companies in the consulting engineering sector and environment, object of this study, have implemented management systems motivated by the need to fulfill a contractual requirement required by the operating companies in the oil sector, rather than to ensure excellent management processes. This research aims to define a methodology for a comprehensive model of management for consulting firms that provide services to firms belonging to the subsector of oil exploitation in Colombia, in order to properly articulate the integrated management systems Health, Safety, Environment, Quality - HSEQ using as the linchpin of social responsibility principles contained in ISO 26000.

An array of variables and hypotheses are considered. Based on the principles of ISO 26000 a survey and a methodology are designed, the latter is made up of three components: strategic, technical/operational and human. Data triangulation is used in order to ensure the validity of the results. It was found that the principles of ISO 26000 are articulating elements within the components of the methodology. As this is an applied research, these results can be implemented in other organizations with integrated HSEQ management systems and RUC.

Keywords: Integral management, HSEQ, RUC, corporate social responsibility, integrated management systems, ISO 26000.

\section{INTRODUCCIÓN}

Esta investigación busca definir una propuesta metodológica para un modelo de gestión integral que favorezca sinergias entre los sistemas integrados de gestión, es decir Health, Safety, Environment and Quality (HSEQ) y el Registro Uniforme de Evaluación del Sistema de Seguridad, Salud en el Trabajo y Protección Ambiental para Contratistas (RUC), que son utilizados por las empresas de consultoría en ingeniería y medioambiente en el sector de hidrocarburos en Colombia.

La implementación de los sistemas de gestión HSEQ y RUC en dichas empresas se hace para cumplir con requisitos licitatorios establecidos por las empresas operadoras, quienes otorgan puntuación adicional en la calificación de las propuestas a quienes demuestren haberlos implementado. Lo anterior, a su vez, mejora la posibilidad de que les sean adjudicados los contratos.

La premura y desarticulación con que se suelen implementar y certificar dichos sistemas de gestión genera problemas, tales como: gestión desarticulada, reprocesos, falta de integración, ineficiencias, y peor aún, al implementar los sistemas de gestión no se obtienen los resultados de gestión esperados. 
Las empresas objeto de estudio pueden mitigar los impactos (ambientales, socioculturales, entre otros) al incorporar en sus prácticas el concepto de responsabilidad social empresarial (RSE), la cual se entiende como una filosofía, una actitud y visión a largo plazo que adopta la empresa hacia los negocios, que se refleja en la incorporación voluntaria en su gestión de las preocupaciones y expectativas de sus distintos grupos de interés (stakeholders). De este modo, la investigación plantea que los principios de ISO 26000 son los elementos articuladores de los sistemas integrados de gestión HSEQ-RUC. Se escogió ISO 26000 entre los demás estándares de RSE por la importancia que otorga a la participación del personal y partes interesadas de la organización, además porque es compatible con otros sistemas de gestión.

Tejada y Peña sugieren que la gestión integral de las organizaciones, consideradas sistemas abiertos, guarda relación con los aspectos internos (integración y articulación entre el direccionamiento estratégico, la estructura del negocio, los sistemas de gestión y las culturas de la organización), así como con las posibilidades que ofrece para el Desarrollo Sostenible ${ }^{1}$.

Así mismo, consideran que los principios de ISO 26000 son los elementos articuladores entre los sistemas integrados HSEQ - RUC. Sin embargo, implementar dichos sistemas solo como respuesta a requisitos del mercado, podría desvirtuar su enfoque dentro de la administración de los riesgos en las empresas, debido a que no se enlazan con la realidad de la empresa y solo existen en el papel. En este sentido, la competencia y el incremento en la participación del personal en las actividades relacionadas con el desarrollo de los sistemas integrados de gestión HSEQ - RUC son factores esenciales que facilitan la articulación entre dichos sistemas.

\section{METODOLOGÍA}

La investigación es aplicada y comprensiva/interpretativa. Tomando como punto de partida los principios de ISO 26000, denominados categorías en esta investigación, se comparan los elementos comunes entre los sistemas integrados HSEQ-RUC y se realiza el despliegue de los principios en sub-categorías, variables y elementos a indagar (ver figura 1 Metodología empleada para la definición de variables). Estos últimos se reflejan en las preguntas de las encuestas aplicadas en las empresas de la muestra representativa. De otra parte, el análisis de los elementos comunes entre los sistemas integrados HSEQ-RUC sirven para el diseño de la Propuesta Metodológica del Modelo de Gestión Integral con su respectiva guía de implementación.

Figura 1. Metodología empleada para la definición de variables

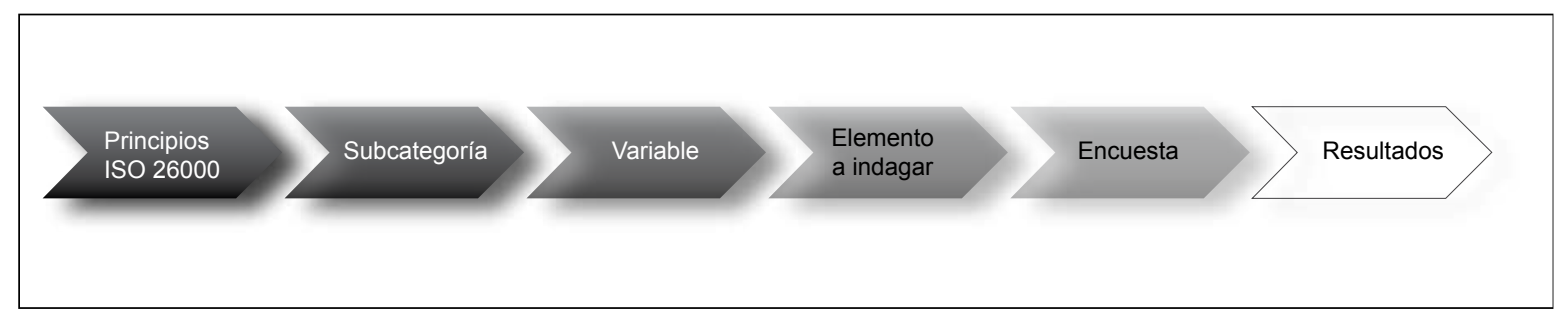

Fuente: elaboración propia

1 TEJADA L, Fabio y PEÑA G, Guillermo. Reflexiones sobre las características constitutivas de la gestión integral. En: Revista Signos. Bogotá: Universidad Santo Tomás. Noviembre de 2009, p. $79-93$. 
Los principios de responsabilidad social que establece la Guía ISO 26000² se describen a continuación:

- Rendición de cuentas: este principio propone que una organización debería aceptar el escrutinio adecuado de sus partes interesadas y responder a ese escrutinio. Implica también la obligación de la Dirección de responder ante los propietarios o accionistas y por parte de la organización de responder ante las autoridades legales en relación con las leyes y regulaciones.

- Transparencia: una organización debería revelar de forma clara, precisa y completa y en un grado razonable y suficiente la información sobre las políticas, decisiones y actividades de las que es responsable, incluyendo sus impactos conocidos y probables sobre la sociedad y el medio ambiente. Esta información debería estar fácilmente disponible y ser directamente accesible y entendible para las partes interesadas.

- Comportamiento ético: el comportamiento de una organización debería basarse en los valores de la honestidad, equidad e integridad. Estos valores implican la preocupación por las personas, animales y medio ambiente, y un compromiso de abordar el impacto de sus actividades y decisiones en los intereses de las partes interesadas.

- Respeto a los intereses de las partes interesadas: una organización debería respetar, considerar y responder a los intereses de sus partes interesadas. Para ello debe identificarlas, reconocer y mostrar el debido respeto por sus intereses y derechos legales y responder a las inquietudes que manifiesten.

2 ISO, Norma Internacional ISO 26000, Guía de Responsabilidad Social, primera edición 2010-11-01. p. 11-15
- Respeto al principio de legalidad/Estado de Derecho: el respeto al principio de la legalidad se refiere a la supremacía del derecho y, en particular, a la idea de que ningún individuo u organización está por encima de la ley y de que los gobiernos también están sujetos a la ley.

- Respeto a la normativa internacional de comportamiento: una organización debería respetar la normativa internacional de comportamiento, a la vez que acatar el principio de respeto al principio de legalidad.

- Respeto a los derechos humanos: una organización debería respetar los derechos humanos y reconocer, tanto su importancia como su universalidad.

Si bien ISO 26000 plantea siete principios, en la investigación se excluye el principio de Respeto a la normativa internacional de comportamiento, debido a que no se considera aplicable a las empresas objeto de estudio. Además, sus actividades e impactos se encuentran enmarcados en las obligaciones contractuales establecidas con la operadora, las cuales son reglamentadas por la ley nacional. Por esta razón se decide no incluir en esta investigación dicho principio, por considerar además que no aporta información adicional.

Dado que la investigación adopta el concepto de gestión integral de Tejada y Peña ${ }^{3}$, también se incluyeron en el proceso de definición de las variables de investigación los componentes de la gestión interna definidos por dichos autores: direccionamiento estratégico, estructura del negocio, sistemas de gestión y culturas de la organización, articulado con los principios de responsabilidad social definidos en ISO 26000.

3 TEJADA L, Fabio y PEÑA G, Guillermo. Reflexiones sobre las características constitutivas de la gestión integral. En: Revista Signos. Bogotá: Universidad Santo Tomás, Noviembre de 2009, pp. 79-93. 
El enfoque de la investigación es mixto con predominio cualitativo, pues se aborda inicialmente con información estadística para conocer el diagnóstico de condiciones iniciales. Luego, mediante encuestas, se realiza la identificación y el planteamiento de unos elementos articuladores entre ambos HSEQ-RUC y RSE los cuales se proponen y validan. En el diseño de la encuesta se definen unos niveles de madurez, calificados cuantitativa y cualitativamente (ver Cuadro 1. Descripción de las escalas de calificación).

Cuadro 1. Descripción de las escalas de calificación

\begin{tabular}{|c|c|c|}
\hline CALIFICACIÓN & $\begin{array}{l}\text { DESCRIPCIÓN DE } \\
\text { LA CALIFICACIÓN }\end{array}$ & $\begin{array}{l}\text { NIVEL DE } \\
\text { MADUREZ }\end{array}$ \\
\hline $\begin{array}{l}\text { MENOR } 0 \\
\text { IGUAL A } 3\end{array}$ & $\begin{array}{l}\text { La empresa se caracteriza } \\
\text { por un desarrollo parcial de } \\
\text { los requisitos definidos } \\
\text { en los referenciales HSEQ } \\
\text { y RUC. No hay planeación } \\
\text { ni metas establecidas y las } \\
\text { acciones se realizan } \\
\text { de manera desarticulada. }\end{array}$ & Existe \\
\hline $\begin{array}{l}\text { ENTRE } 3.01 \\
\quad \text { Y } 4.5\end{array}$ & $\begin{array}{l}\text { Las acciones realizadas por } \\
\text { la empresa tienen un mayor } \\
\text { grado de articulación con } \\
\text { los sistemas HSEQ y RUC. } \\
\text { Los colaboradores conocen } \\
\text { dichos sistemas y realizan } \\
\text { su trabajo acorde con } \\
\text { los mismos. }\end{array}$ & Apropiación \\
\hline MAYOR A 4.5 & $\begin{array}{l}\text { La empresa involucra la lógica } \\
\text { del mejoramiento continuo: } \\
\text { evalúa sus procesos y resultados } \\
\text { y, en consecuencia, los } \\
\text { ajusta y mejora. }\end{array}$ & $\begin{array}{l}\text { Mejora } \\
\text { Continua }\end{array}$ \\
\hline
\end{tabular}

Fuente: elaboración propia.

La muestra sobre la cual se desarrolló este trabajo de investigación la constituyen 11 de las $131^{4}$ empresas consultoras de ingeniería y medio ambiente de Bogotá

4 Estadísticas tomadas con referencia en la base de datos del área comercial de Anteagroup, estudio de mercado realizado para el segundo semestre de 2013, con corte a Junio 30 de 2013, suministrado por el depto. de mercadeo. que prestan sus servicios a empresas del subsector de la explotación de petróleo en Colombia. Estas empresas cuentan con sistemas integrados HSEQ (implementados y certificados), además, están certificadas por el Consejo Colombiano de Seguridad en el Registro Uniforme de Evaluación del Sistema de Gestión en Seguridad, Salud Ocupacional y Ambiente - RUC y cuentan con una trayectoria superior a los 10 años en el mercado.

Sin embargo, de las 11 empresas contactadas, solo 4 de ellas responden la encuesta. La prueba piloto para validar la propuesta se hizo en la empresa Anteagroup, una de las empresas objeto de estudio.

Con el fin de garantizar la validez y confiabilidad de la investigación se usa la triangulación. Inicialmente se realiza una revisión bibliográfica para establecer los elementos comunes en los sistemas de gestión analizados y definir las variables de investigación. Posteriormente se comparan los principios de ISO 26000, los requisitos de las normas ISO 9001 Sistemas de Gestión de la Calidad, ISO 14001 Sistemas de Gestión Ambiental y OHSAS 18001 Sistemas de Gestión en Seguridad y Salud Ocupacional, así como los componentes de la gestión interna definidos por Tejada y Peña ${ }^{5}$ (direccionamiento estratégico, estructura del negocio, sistemas de gestión y culturas de la organización). Durante este proceso se identifican los elementos comunes entre los sistemas integrados de gestión HSEQ y RUC, y se ubican dichos principios en subcategorías, variables y elementos a indagar.

Con estos últimos se elabora la encuesta que se aplica en la muestra de empresas para conocer sus percepciones acerca de las variables de investigación, y finalmente se realiza la validación de la propuesta metodológica mediante una prueba piloto.

Para pasar de la integración de sistemas de gestión a la gestión integral a partir de los resultados consolidados

5 TEJADA L, Fabio y PEÑA G, Guillermo. Óp. Cit. pp. 79-93. 
de la aplicación de la encuesta, construida a partir de los principios de ISO $26000 \mathrm{y}$ del análisis documental previo, se diseña un modelo de gestión integral que busca fortalecer el componente humano de las organizaciones y facilitar el logro de sus objetivos.

La Propuesta Metodológica del Modelo de Gestión Integral tiene tres componentes: estratégico, técnico/ operativo y humano. Así mismo, cada componente tiene su propio ciclo: Planear, Hacer, Verificar y Actuar - PHVA. Para validar la propuesta metodológica se elabora una ruta de implementación, la cual está asociada a cada elemento clave, a cada componente y a cada etapa del ciclo PHVA.

La prueba piloto se desarrolla en una empresa utilizando la metodología propia de la consultoría. Allí, mediante sesiones de trabajo, se asignan acciones y actividades para implementar la propuesta metodológica del modelo de gestión, y simultáneamente, se lleva a cabo un seguimiento y control. Esta prueba permite validar la propuesta metodológica del modelo de gestión y la necesidad de realizar ajustes para su puesta en práctica.

\section{RESULTADOS Y DISCUSIÓN}

La aplicación de las encuestas permite evidenciar que las empresas del sector se encuentran en el nivel de madurez denominado "Apropiación”, es decir, un nivel medio de madurez para las variables de investigación definidas, a excepción de la variable "Identificación de Requisitos Legales" de la sub-categoría denominada "Planeación del control operacional", la cual se encuentra en nivel "Existe". Es decir, en un nivel bajo de madurez" (cuadro 2). En términos generales, los resultados de la percepción de los encuestados evidencian que en las empresas analizadas se trabaja conforme a los sistemas HSEQ-RUC. Además, evidencian que existen oportunidades de mejora para pasar al nivel de madurez de Mejora Continua, en el cual existe una mayor apropiación de los sistemas de gestión por parte del personal y de la Alta Dirección. Esta situación pone de manifiesto la importancia de la participación del personal para mejorar la eficacia de los sistemas de gestión en las organizaciones.

El diseño de la propuesta metodológica se justificó desde dos puntos de vista: 1) la identificación de elementos comunes entre los principios de ISO 26000, los requisitos de los sistemas de gestión HSEQ y RUC y los componentes de la gestión interna definidos por Tejada y Peña ${ }^{6}$. 2) Los resultados del diagnóstico de gestión en las empresas del sector objeto de estudio. Debido a que el $80 \%$ o más de las respuestas obtenidas en cada una de las variables de investigación fueron concluyentes se pudo evidenciar que los elementos, variables, sub categorías y categorías de investigación están articulados, y además, que dichas variables son la base que permiten el diseño de la propuesta metodológica.

La propuesta metodológica tiene tres componentes: estratégico, técnico/operacional y humano. El componente estratégico está integrado por el direccionamiento estratégico, los procesos y componentes que integran la gobernanza de la organización, los procesos de comunicación interna y externa de la organización y el tablero de mando con sus respectivos indicadores de gestión. El componente operativo técnico lo constituyen los procesos y actividades que desarrollan las organizaciones para ofrecer sus productos y servicios, al igual que los mecanismos de seguimiento y control que permiten evidenciar el cumplimiento de los objetivos esperados por todas las partes interesadas de la organización. Y el componente humano está relacionado con las actividades, procesos, programas, proyectos y acciones desarrolladas con el fin de mejorar el compromiso, conciencia y creatividad de las personas de la organización; quienes apoyan y apalancan de forma transversal las actividades y procesos que se desarrollan al interior de las organizaciones.

6 Ibíd. 
Cuadro 2. Resultados consolidados del sector

\begin{tabular}{|c|c|c|c|c|}
\hline $\begin{array}{c}\text { PRINCIPIO } \\
\text { DEL MODELO DE } \\
\text { RESPONSABILIDAD } \\
\text { SOCIAL } \\
\text { EMPRESARIAL } \\
\text { RSE (ISO 26000) }\end{array}$ & SUBCATEGORÍA & VARIABLE & $\begin{array}{l}\text { CALIFICACIÓN } \\
\text { DEL SECTOR }\end{array}$ & $\begin{array}{l}\text { NIVEL } \\
\text { DE } \\
\text { MADUREZ }\end{array}$ \\
\hline \multirow{2}{*}{$\begin{array}{l}\text { RENDICIÓN } \\
\text { DE CUENTAS }\end{array}$} & Balance Social & Indicadores de Gestión & 3,86 & Apropiación \\
\hline & $\begin{array}{l}\text { Identificación y análisis de necesidades y } \\
\text { requisitos. }\end{array}$ & Reportes & 3,3 & Apropiación \\
\hline TRANSPARENCIA & Apertura al escrutinio & Apertura al escrutinio & 3.81 & Apropiación \\
\hline $\begin{array}{l}\text { COMPORTAMIENTO } \\
\text { ÉTICO }\end{array}$ & $\begin{array}{l}\text { "Direccionamiento Estratégico(la misión, } \\
\text { visión, los valores, principios y código de } \\
\text { conducta de la propia organización) } \\
\text { Políícas de la organización } \\
\text { (estructuras para la toma de decisiones } \\
\text { en la organización y naturaleza } \\
\text { de la toma de decisiones)" }\end{array}$ & $\begin{array}{l}\text { Objetivos y metas } \\
\text { de la organización }\end{array}$ & 3,98 & Apropiación \\
\hline \multirow{3}{*}{$\begin{array}{c}\text { RESPETO } \\
\text { A LOS INTERESES } \\
\text { DE LAS PARTES } \\
\text { INTERESADAS }\end{array}$} & $\begin{array}{l}\text { Gobernanza de la organización } \\
\text { (estructuras para la toma de decisiones } \\
\text { en la organización y naturaleza } \\
\text { de la toma de decisiones) }\end{array}$ & $\begin{array}{l}\text { Identificación de estructura } \\
\text { organizacional, roles, } \\
\text { responsabilidad y autoridad. }\end{array}$ & 4,4 & Apropiación \\
\hline & Sistemas de gestión integrados & $\begin{array}{l}\text { Enfoque preventivo o correctivo } \\
\text { de los sistemas de gestión } \\
\text { integrados }\end{array}$ & 3.72 & Apropiación \\
\hline & Cadena de valor de la organización & Portafolio de productos y servicios & 4.00 & Apropiación \\
\hline \multirow{3}{*}{$\begin{array}{l}\text { RESPETO AL } \\
\text { PRINCIPIO } \\
\text { DE LEGALIDAD }\end{array}$} & Planeación del control operacional & Identificación de requisitos legales & 2.85 & Existe \\
\hline & Control Operacional & $\begin{array}{l}\text { Mecanismos de seguimiento } \\
\text { y medición }\end{array}$ & 3.61 & Apropiación \\
\hline & $\begin{array}{l}\text { Evaluación de cumplimiento } \\
\text { legal y contractual }\end{array}$ & Revisión por la dirección & 4.10 & Apropiación \\
\hline \multirow{4}{*}{$\begin{array}{c}\text { RESPETO A LOS } \\
\text { DERECHOS HUMANOS }\end{array}$} & \multirow[t]{3}{*}{ Derechos y deberes de los empleados } & Ambiente de trabajo & 4.35 & Apropiación \\
\hline & & $\begin{array}{l}\text { Derechos y deberes } \\
\text { de los empleados }\end{array}$ & 3.87 & Apropiación \\
\hline & & Procesos de comunicación interna & 3.80 & Apropiación \\
\hline & $\begin{array}{l}\text { Competencia del personal } \\
\text { (características de la mano de obra } \\
\text { o de los empleados de la organización, } \\
\text { incluyendo la mano de obra } \\
\text { contratada) }\end{array}$ & $\begin{array}{l}\text { Crecimiento y Desarrollo } \\
\text { profesional }\end{array}$ & 3.80 & Apropiación \\
\hline
\end{tabular}

Fuente: elaboración propia. 
En cada componente están presentes los principios de ISO 26000 como elementos articuladores, situación que se denominó "Ciudadanía sistémica de los principios de ISO 26000 en la propuesta metodológica de un modelo de gestión integral". (Ver Figura 2).

Figura 2. Ciudadanía sistémica de los principios de ISO 26000 en la propuesta metodológica de un modelo de gestión integral

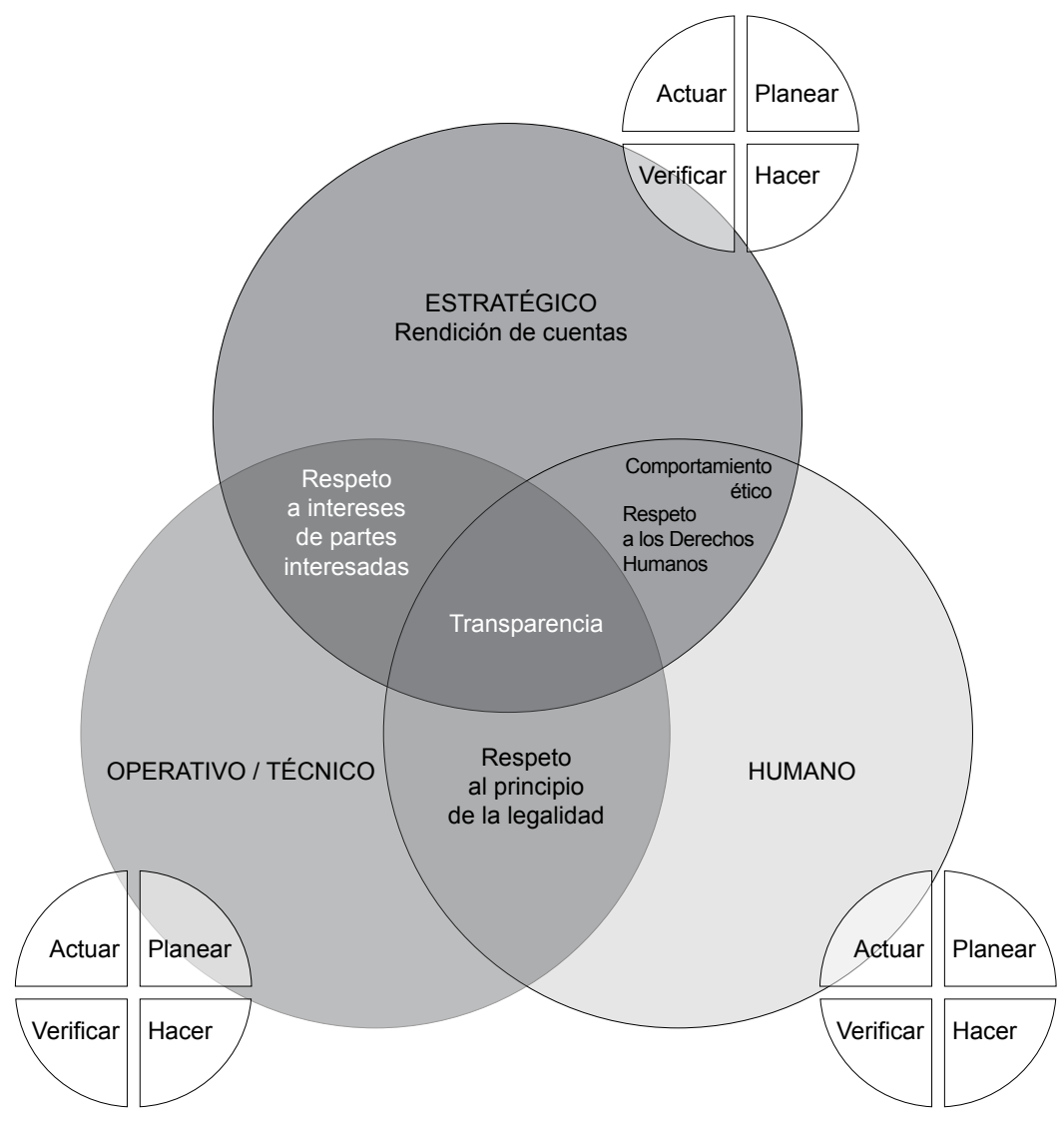

Fuente: elaboración propia

a. El principio de Transparencia de ISO 26000 es el eje articulador de los tres componentes definidos (estratégico, humano y técnico/operativo). De este modo, la transparencia se podría definir como la ciudadanía sistémica de la propuesta metodológica del modelo de gestión integral.

b. El principio de respeto ante los intereses de las partes interesadas articula los componentes estratégico y el técnico/operativo. Con esto se concluye que a través de este principio se mejora la participación activa y el desarrollo de la comunidad.

c. Los principios de comportamiento ético y respeto a los derechos humanos, articulan dos componentes: estratégico y humano. La definición de políticas y valores de una empresa como directrices generadas por la dirección orientan la realización de buenas prácticas laborales que "comprenden todas las políticas y prácticas relacionadas con el trabajo 
en nombre de la Organización, incluido el trabajo subcontratado", 7 y también las prácticas justas de operación, "la competencia justa y el respeto a los derechos de propiedad no se pueden lograr, si las Organizaciones no operan entre ellas de manera honesta, equitativa e íntegra". ${ }^{8}$

d. El principio de respeto al principio de legalidad articula el componente técnico y el humano. Este principio permite realizar un adecuado control operacional de los sistemas integrados HSEQ-RUC y de todas las actividades que ejecuta la organización (incluyendo el respeto por los derechos y deberes de los empleados), tomando en consideración que es el componente humano el factor que apalanca y genera valor agregado en la gestión de las empresas.

e. Por último, el principio de rendición de cuentas se encuentra al interior del componente estratégico, en este sentido, hace parte de las responsabilidades y obligaciones legales que tiene un representante legal (i.e cumplir con todos los requisitos de información legal, contractual entre otros).

$\mathrm{Al}$ interior de cada componente se desarrolla el ciclo Planear, Hacer, Verificar y Actuar - PHVA (ver figuras $3,4$ y 5$)$.

Figura 3. Ciclo PHVA Componente estratégico

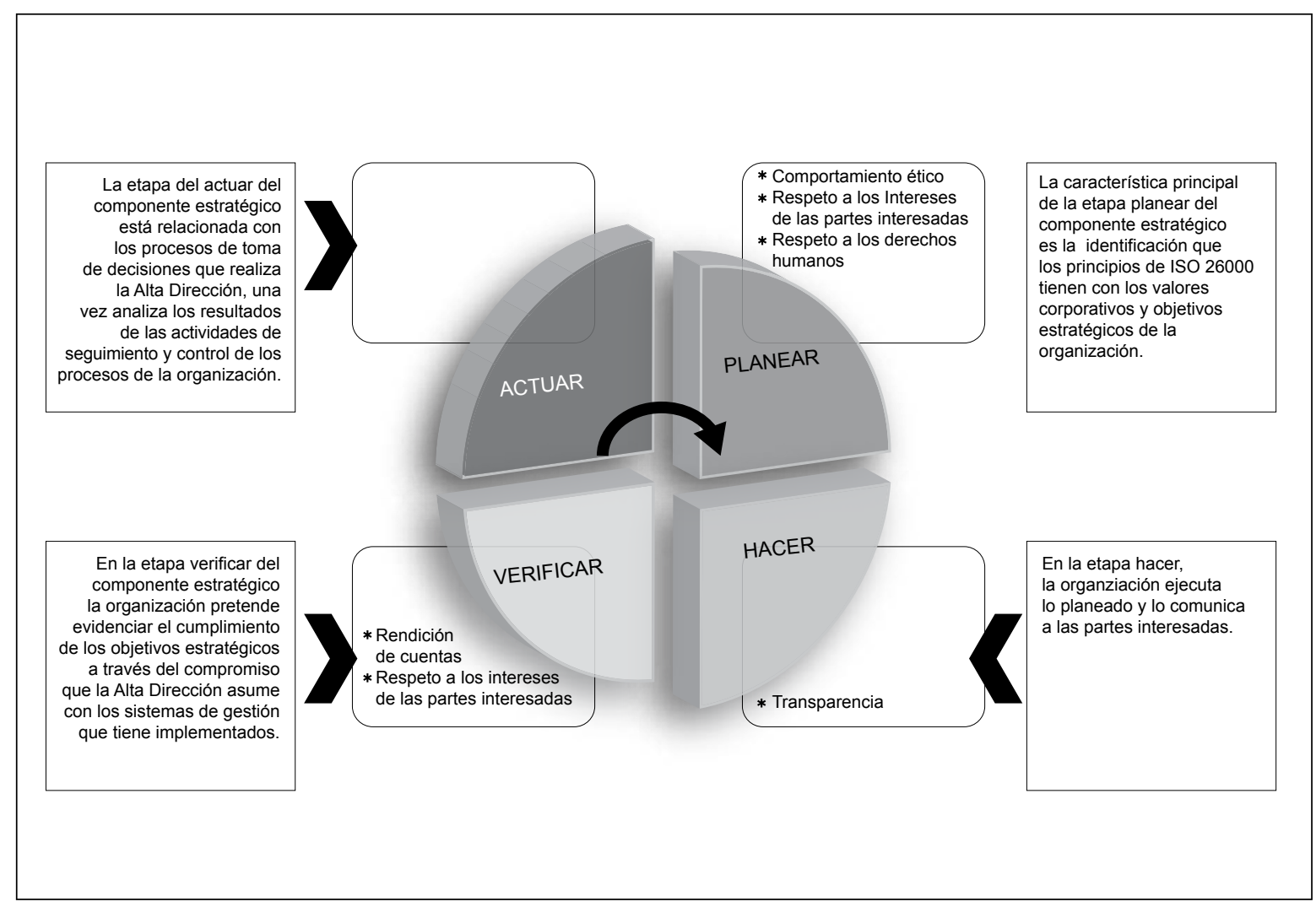

7 ROMERO, Miguel A. Nueva Norma ISO 26000:2010 "Guía de responsabilidad social”. Óp. Cit. p. 6

8 Ibíd. p. 7 
Ciclo PHVA Componente Estratégico: El ciclo de mejora continua o PHVA del componente estratégico es liderado por la Alta Dirección de las Organizaciones. La planeación del componente estratégico está relacionada con roles y actividades propias de la Alta Dirección, tales como la definición o revisión del direccionamiento estratégico, los cambios en la estructura de toma de decisiones, los productos y servicios de la organización, recursos, planes de acción, entre otros. La ejecución o el hacer del componente estratégico son todas las actividades que sirven de soporte para la puesta en marcha o implementación de lo planeado. La verificación del componente estratégico incluye aquellas actividades donde se evidencia que la organización está cumpliendo con los objetivos estratégicos a través del compromiso que la Alta Dirección asume con los sistemas de gestión que tiene implementados. Por último, el actuar está relacionado con los procesos de toma de decisiones que realiza la Alta Dirección, una vez analiza los resultados de las actividades de seguimiento y control de los procesos de la organización, así como las decisiones que conduzcan a la mejora de los resultados de gestión de la organización.

Fiǵura 4. Ciclo PHVA Componente técnico/operativo

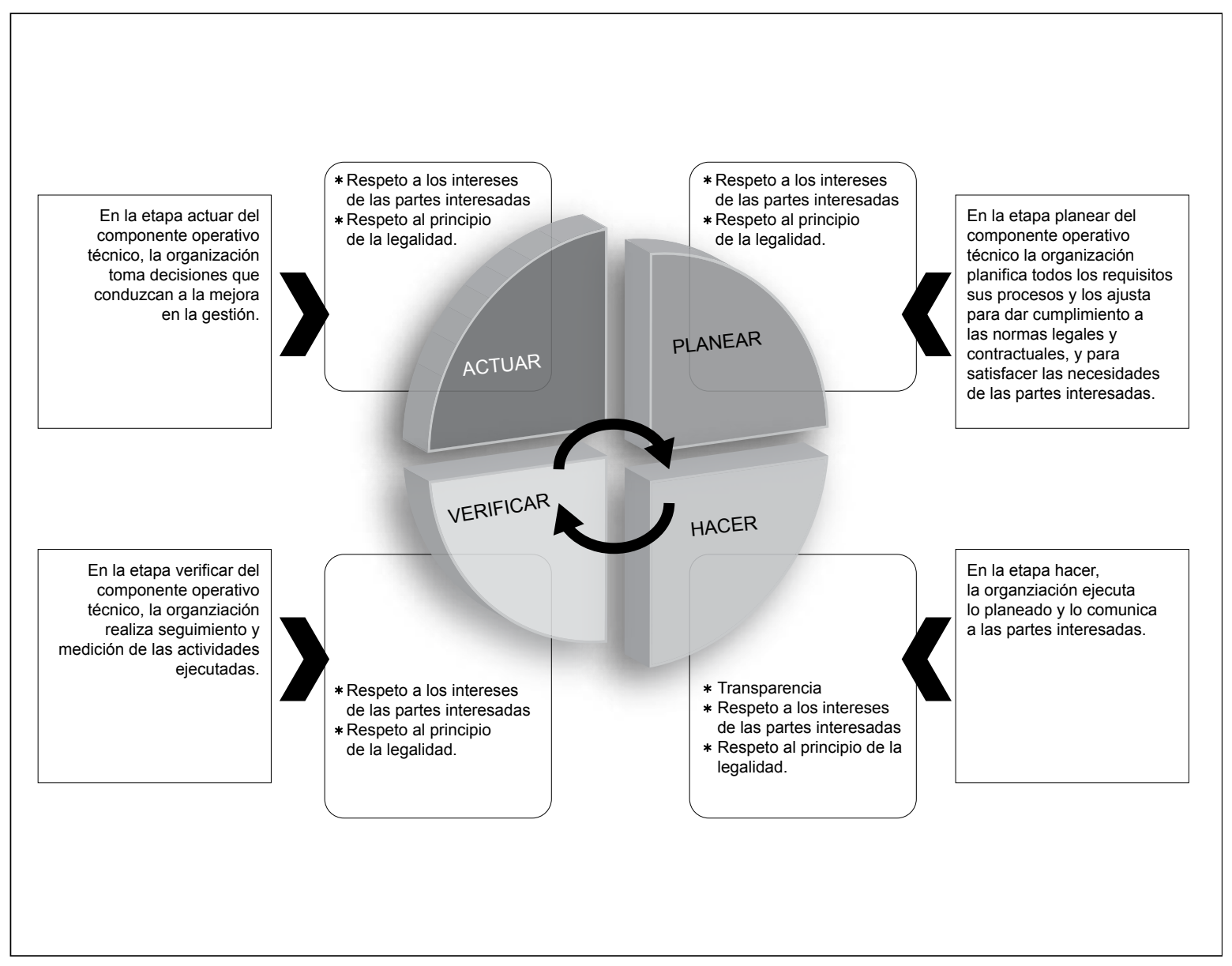


Ciclo PHVA Componente técnico/operativo: El ciclo de mejora continua $o$ PHVA del componente operativo técnico es liderado por los líderes del proceso de los sistemas de gestión que operan al interior de la organización. La planeación del componente Operativo/ Técnico se debe realizar al menos una vez al año, y en ella definir o revisar el método de trabajo, realizar la planeación del control operacional, definir o actualizar los requisitos de calidad del cliente, del sector y establecer la capacidad operativa. La ejecución 0 el hacer del componente Operativo/Técnico incluye todas las actividades que sirven de soporte para la puesta en marcha o implementación de lo planeado. En la etapa verificar del componente operativo técnico, la organización realiza seguimiento y medición de las actividades ejecutadas a través de auditorías internas, revisión por parte de la dirección, entre otros. En la etapa actuar del componente operativo técnico la organización toma decisiones que conduzcan a la mejora de la gestión.

Figura 5. Ciclo PHVA Componente humano

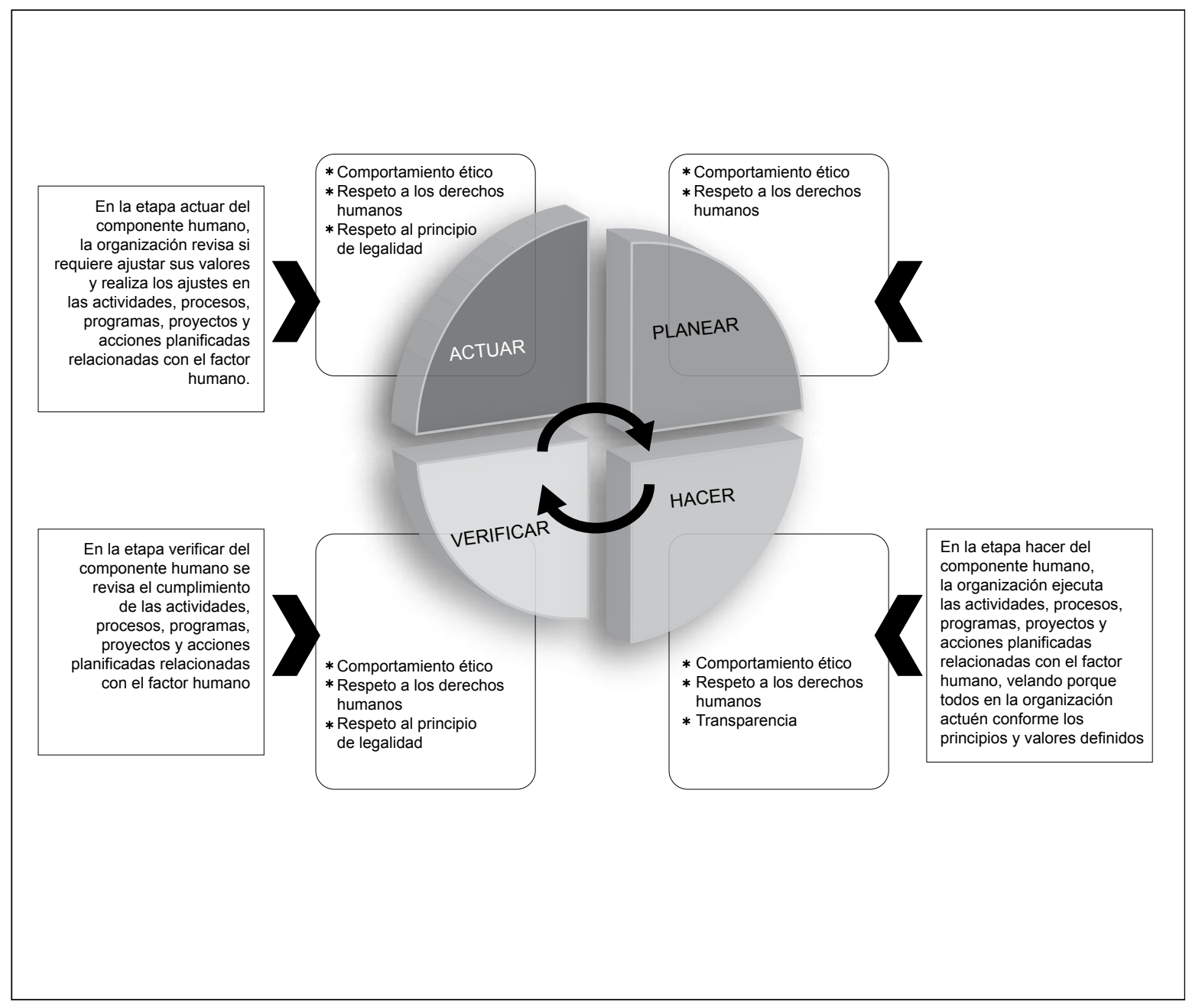


Ciclo PHVA Componente humano: El ciclo de mejora continua o PHVA del componente humano es liderado por la Alta Dirección o el líder de talento humano de la organización. En la etapa de planeación del Componente Humano la organización define y planifica las actividades, procesos, programas, proyectos y acciones cuyo objetivo y alcance lo constituye el factor humano. En el hacer del componente humano la organización ejecuta las actividades, procesos, programas, proyectos y acciones planificadas relacionados con el factor humano. En este componente se vela porque todos en la organización actúen de acuerdo a los principios y valores definidos. En la etapa verificar del componente humano se revisa el cumplimiento de las actividades, procesos, programas, proyectos y acciones planificadas relacionados con el factor humano; es clave en esta etapa evaluar el clima organizacional y el desempeño del personal. En la etapa actuar del componente humano la organización revisa si requiere ajustar sus valores, actividades, procesos, programas, proyectos y acciones planeadas que se encuentren relacionadas con el factor humano, tomando como referente el seguimiento y control, con el fin de mejorar el desempeño de este componente.

Para implantar la propuesta metodológica se elaboró una metodología de implementación, a modo de guía, que contiene las etapas, acciones y actividades. La metodología plantea que la implementación del modelo se debe realizar de acuerdo al ciclo de la mejora continua - PHVA de cada uno de sus tres componentes: estratégico, operativo/técnico y humano - teniendo en cuenta las actividades, metodologías de trabajo y los resultados esperados que son propios de cada etapa del ciclo, con el fin de que se observe coherencia con la estructura y funcionamiento de cada componente. La metodología tiene las siguientes etapas: a. Diagnóstico de la organización con respecto a la propuesta metodológica.

b. Sensibilización de la organización con respecto a la propuesta metodológica.

c. Planeación de implementación de la propuesta metodológica.

d. Realización de prueba piloto de la propuesta metodológica.

e. Evaluación de los resultados.

f. Definición de acciones de mejora para la propuesta metodológica.

Tanto la planeación de la implementación como la realización de la prueba piloto deben contar con una alta participación del personal, en especial del nivel directivo, además de las partes interesadas de la organización, con el fin de mejorar la apropiación y sostenibilidad de la propuesta metodológica y obtener los resultados de forma eficaz y oportuna.

Se realizó la prueba piloto y la validación de la propuesta metodológica del modelo de gestión integral en una empresa de la muestra representativa. La investigación permite concluir que los resultados no solo pueden aplicarse a empresas similares a las definidas como objeto de estudio, sino a aquellas que cuenten con los sistemas integrados HSEQ-RUC implementados.

Los resultados de la prueba piloto realizada evidenciaron lo siguiente:

a. Las acciones y actividades para implementar la propuesta metodológica son realizables, pues no requieren de inversión de recursos. Por ejemplo, algunas acciones ya se estaban ejecutando de forma desordenada, sin embargo, a partir de la prueba piloto se incorporaron y alinearon con el sistema de gestión integral. Estas acciones y actividades son 
fáciles de medir ya que la línea base está dada por los resultados de las auditorias internas, sin embargo, requieren del compromiso tanto de la Alta Dirección como del personal de la organización que lidera los elementos claves y acciones identificadas.

b. Las acciones de la propuesta metodológica que implican la inversión de recursos generan algo de resistencia por parte de la Alta Dirección, incluso en aquellas cuyo nivel de madurez en los elementos del modelo se encuentre en nivel de "Apropiación".

c. A través de la implementación de la propuesta metodológica, la organización identificó nuevas herramientas para realizar planeación del control operacional. En particular este aspecto fue calificado en el nivel 'Existe'. Se identificó que varios de los proyectos en desarrollo pueden aportar al control operacional, para pasar al nivel de 'Apropiación', y a su vez evidenciar mejoras que faciliten el control operacional.

d. Se identifican temas y aspectos importantes en los cuales se requiere capacitar al personal y estandarizar metodologías, tal como la administración de riesgos.

e. Dentro del componente humano se implementaron acciones que buscan mejorar la planeación de la cultura organizacional. Entre ellas aparecen: la actualización del reglamento interno de trabajo, los perfiles de ingreso a la organización, los planes de capacitación y bienestar que mitigan el riesgo de rotación del personal. Igualmente se implementaron acciones para garantizar que a todo el personal de la organización se le entregaran por escrito sus funciones.

f. Se socializó el protocolo y la herramienta para la evaluación del desempeño de los empleados, y se definió como insumo para actualizar los planes de capacitación de la organización.

\section{CONCLUSIONES}

Se elaboraron tres herramientas con una estructura similar y coherente para realizar el diagnóstico, la planeación de la implementación y la prueba piloto de la propuesta metodológica de un sistema de gestión integral. En la estructura de las herramientas se definieron elementos claves para cada ciclo de cada componente de la propuesta metodológica. Así mismo, para cada elemento clave se describieron las características asociadas que debía tener con los tres niveles de madurez, las cuales, a su vez, son consistentes con los criterios establecidos para interpretar los resultados de la encuesta aplicada. Esta estrategia permitió conservar coherencia en las actividades realizadas desde el diagnóstico hasta la realización de la prueba piloto.

A partir de los resultados de la investigación, tanto en la revisión documental como en la aplicación de la encuesta y la prueba piloto, es evidente que los principios de ISO 26000 pueden servir como ejes articuladores para la gestión integral en las organizaciones, según la relación entre cada principio y los componentes de la gestión integral:

- Rendición de cuentas: está inmerso en el componente estratégico de la propuesta metodológica, dado que la Alta Dirección tiene como responsabilidad y obligación legal rendir cuentas de la gestión realizada, cumpliendo con todos los requisitos de orden legal, contractual, procedimental, entre otros. Se vuelve operativo a través de actividades de los sistemas integrados HSEQ-RUC, como por ejemplo, la revisión por parte de la dirección.

- Transparencia:es la ciudadanía sistémica de la propuesta metodológica que agrupa los tres componentes, dado que solo mediante los procesos de comunicación interna y externa con las partes interesadas se favorece la apropiación de los sistemas integrados de gestión HSEQ-RUC, y se realiza una mejor gestión del cambio. 
- Comportamiento ético y Respeto a los derechos humanos: hace parte de los componentes Estratégico y Humano. La definición del direccionamiento estratégico, planes de la organización, el código de ética y demás normas de convivencia caracterizan el componente estratégico.

- Respeto a los intereses de las partes interesadas: está contenido en los componentes Estratégico y Técnico/operativo. La Alta Dirección y los líderes de los sistemas integrados HSEQ-RUC deben incluir como insumos de la planeación de sus actividades y procesos los requisitos legales, ambientales, de calidad, de seguridad y salud ocupacional, y a todos aquellos que generen un impacto o afectación a las partes interesadas. Este componente abarca todas las etapas del ciclo PHVA del componente técnico/operativo, así como el planear y actuar del componente estratégico, dado que la definición de los planes de acción, la asignación de recursos, el establecimiento de nuevos productos o servicios, están orientados al cumplimiento de los objetivos de la organización.

- Respeto al principio de legalidad: está incluido en los componentes Técnico/operativo y Humano, pues según el direccionamiento estratégico, los líderes de los sistemas integrados HSEQ-RUC incluyen el aspecto legal en la definición de los requisitos que consideran la planeación, operación, seguimiento y control de los procesos de la organización. Estos requisitos también incluyen la determinación de aspectos legales relacionados con la vinculación y desarrollo del factor humano. Este componente se asocia con todas la etapas del ciclo PHVA del componente Técnico/Operativo, y con la verificación y actuación del componente humano de la propuesta.

Cada uno de los componentes de la propuesta metodológica cuenta con un ciclo de mejora continua en etapas de Planear, Hacer, Verificar y Actuar - PHVA que se articula con los principios de la Responsabilidad Social. La investigación permitió evidenciar que existen principios de ISO 26000 que hacen parte de la gestión de uno o más componentes del modelo de gestión integral propuesto. En este punto de la investigación se retoma el concepto de ciudadanía sistémica formulado por Edgar Morin. Bajo esta premisa se hace referencia al concepto de ciudadanía sistémica de los principios de ISO 26000 en la propuesta metodológica del modelo de gestión integral donde la transparencia es el eje articulador de los tres componentes. Se resalta que cuando una organización es transparente "respecto a las decisiones y actividades que afectan a la sociedad, la economía y el medio ambiente,"9 se puede afirmar que asume prácticas justas de operación.

Para realizar el diagnóstico de la organización "Anteagroup" con respecto a la propuesta metodológica del modelo de gestión integral se incluyeron, además de la calificación del nivel de madurez de los elementos clave, los resultados de la encuesta, los indicadores de los procesos alcance de la prueba piloto Gestión Estratégica y Gestión de Mejoramiento Organizacional, así como los resultados de las respectivas auditorías internas de estos procesos. Estos resultados permitieron hacer un promedio simple con el cual se concluye que el nivel de madurez de cada componente se encuentra en nivel de "apropiación".

De acuerdo al nivel de madurez se identificaron las acciones requeridas para implementar la propuesta metodológica con la herramienta 'Matriz de Implementación Propuesta Metodológica'. Dichas acciones se implementaron mediante el uso de la metodología propia de la consultoría, es decir, mediante sesiones de trabajo se asignaron unas acciones y actividades a los participantes

9 ROMERO, Miguel A. Nueva Norma ISO 26000:2010 "Guía de responsabilidad social". Global Standards Certification. Disponible en internet http://www.globalstd.com/pdf/rs-iso26000-2010.pdf (Con acceso 21-10-13) 
y posteriormente se realizó seguimiento y control al cumplimiento de dichas actividades.

Los resultados de la prueba piloto permitieron concluir lo siguiente:

a. A partir de la validación de la propuesta metodológica, y de su metodología de implementación, se concluyó que no se requieren realizar ajustes. Lo anterior es debido a que se cumplieron los criterios de confiabilidad de la prueba piloto: por ejemplo, el tiempo y actividades para realizar las acciones se ajustó al cronograma, además, en dichas actividades participaron más del $80 \%$ del personal de los dos procesos definidos para la prueba, y no se observaron oportunidades de mejora para ajustar la propuesta metodológica.

a. Los principios de ISO 26000, al estar inmersos en la operación de los sistemas integrados de gestión HSEQ-RUC, facilitan su articulación, sinergias y evitan reprocesos. Se identificaron actividades que podían ser abordadas de forma transversal, o bien lideradas por un actor con participación de los demás involucrados. Con este resultado particular se comprobó la primera hipótesis de investigación.

b. La implementación de la propuesta metodológica facilita la gestión integral de la organización. Así mismo, permite identificar nuevas herramientas para planear el control operacional, aumentar la eficacia en el seguimiento, en el control de los procesos y en los planes de la organización, los cuales, a su vez, permiten orientar la gestión de la organización hacia la mejora continua.

c. La competencia y aumento de la participación del personal en las actividades relacionadas con el desarrollo de los sistemas integrados de gestión HSEQ - RUC son factores esenciales que facilitan la articulación entre dichos sistemas.

\section{REFERENCIAS}

ISO, Norma Internacional ISO 26000, Guía de Responsabilidad Social (primera edición) 2010. Ginebra. p. 11-15

TEJADA L, Fabio y PEÑA G, Guillermo. Reflexiones sobre las características constitutivas de la gestión integral. En: Revista Signos. Bogotá: Universidad Santo Tomás Noviembre de 2009, pp. 79-93.

ROMERO, Miguel A. Nueva Norma ISO 26000:2010 "Guía de responsabilidad social". Global Standards Certification. Disponible en internet http:// www.globalstd.com/pdf/rs-iso26000-2010.pdf 